Psychotherapeut 2017 $62: 1-2$ DOI 10.1007/s00278-016-0151-y Online publiziert: 22. Dezember 2016 ๑) Springer Medizin Verlag Berlin 2016

CrossMark

\author{
Jörg M. Fegert ${ }^{1} \cdot$ Harald J. Freyberger ${ }^{2}$ \\ ${ }^{1}$ Klinik für Kinder- und Jugendpsychiatrie und Psychotherapie, Universitätsklinikum Ulm, Ulm, \\ Deutschland \\ ${ }^{2}$ Klinik und Poliklinik für Psychiatrie und Psychotherapie, Universitätsmedizin Greifswald, Helios \\ Hanseklinikum Stralsund, Stralsund, Deutschland
}

\title{
Grenzbereich zwischen Jugend- und Erwachsenenalter
}

\section{Psychotherapeutische Aspekte}

Das vorliegende Heft der Zeitschrift Psychotherapeut beschäftigt sich mit psychotherapeutischen Aspekten im Grenzbereich zwischen dem Jugendund dem Erwachsenenalter und greift damit einen bereits 2014 berücksichtigten Themenschwerpunkt wieder auf (Fegert et al. 2014).

Rebecca Groschwitz et al. stellen Daten zu psychiatrischer und psychotherapeutischer Inanspruchnahme anhand einer repräsentativen Allgemeinbevölkerungsstudie vor. Dabei zeigt sich, dass etwa $16 \%$ der Befragten bereits eine Hilfeform in Anspruch genommen haben, die von über $60 \%$ auch als zumindest hilfreich bewertet wird. Die Inanspruchnahmeraten der 14- bis 30-Jährigen und der 31- bis 60-Jährigen liegen etwas höher als bei den über 60-Jährigen. Demgegenüber kann in Kinder- und JugendSamples vermutlich davon ausgegangen werden, dass nur etwa $29 \%$ psychisch Auffälliger einen Behandlungsplatz finden. Über 80 \% der Befragten berichteten eine Wartezeit unter 3 Monaten. Zwischen der Prävalenz psychischer Störungen und der Inanspruchnahme besteht nach wie vor eine gravierende Lücke.

Ulrike Schulze et al. können in einer Studie schwer depressiv erkrankter, stationär behandelter kinder- und jugendpsychiatrischer Patienten zeigen, dass Angstsymptome auf die weitere Psychopathologie und Befindlichkeit erheblichen Einfluss haben und klinisch häufig unterschätzt werden, sodass das Risiko falscher Therapieentscheidungen steigt. So wird ein überzufällig hohes komorbides Auftreten von Ängsten, Zwanghaftigkeit und Alexithymie sowie ein häufiges Vorkommen selbstverletzenden Verhaltens und von Suizidalität in dieser Stichprobe nachgewiesen.

Mehrere Beiträge in diesem Heft beschäftigen sich mit der Transitionsproblematik aus dem Kindes- und Jugend- in das Erwachsenenalter. Jörg M. Fegert et al. berichten in der Rubrik Psychotherapie aktuell über eine Initiative der Deutschen Gesellschaft für Kinderund Jugendpsychiatrie, Psychosomatik und Psychotherapie (DGKJP) und der Deutschen Gesellschaft für Psychiatrie und Psychotherapie, Psychosomatik und Nervenheilkunde (DGPPN), die sich mit der Frage der Transitionspsychiatrie und -psychotherapie befasst hat. Dabei werden die kritischen Hintergrundbedingungen der gegenwärtigen Versorgungssituation dargestellt und Schlussfolgerungen für die Krankenversorgung, die komplementären Versorgungssysteme, die Weiterbildungsordnungen, den Forschungsbedarf und den politischen Handlungsbedarf gezogen.

Einen von ihnen genannten Aspekt greifen Sabine Müller et al. in ihrer für die psychotherapeutische Behandlung relevanten Übersichtsarbeit auf. Sie zeigen, dass das Erreichen der Altersgrenze zur Volljährigkeit für Patienten in kinderund jugendpsychiatrischer bzw. -psychotherapeutischer Behandlung einen gravierenden Einschnitt darstellt, der sich auf die Rechtslage von Zwangsmaßnahmen bzw. auf die Rechtsansprüche auf Hilfen und Versorgungsangebote bezieht. Sie weisen u. a. auf Regelungslücken und "Grauzonen“ hin, die das Risiko implizieren, dass Kinder und Jugendliche - genehmigt durch die Eltern, die sich häufig in einem Interessenkonflikt befinden - massiven Grundrechtseingriffen ausgesetzt sind, ohne dass eine unabhängige Instanz hiervon Kenntnis erlangt und die Rechtfertigung für diese Maßnahmen überprüft.

Armbruster und Freyberger stellen die ersten Arbeitserfahrungen mit einer neu gegründeten Poli- und Tagesklinik für Adoleszenzpsychiatrie und -psychotherapie vor und diskutieren die hierzu notwendigen Rahmenbedingungen, Behandlungselemente und -schwerpunkte sowie kritische Aspekte dieses Ansatzes.

Jörg Fegert schließlich beschäftigt sich mit einem wieder aktuell gewordenen Thema in der Rubrik Psychotherapie aktuell, nämlich dem gerade vorgelegten Eckpunktepapier des Bundesministeriums für Gesundheit zur Novellierung der Ausbildung zum psychologischen Psychotherapeuten und damit mit einem Reformvorhaben, das in dieser Legislaturperiode nicht mehr kommen wird (Bundesministerium für Gesundheit 2016). Obgleich im Forschungsgutachten zur Vorbereitung der Reform (Strauß et al. 2009) explizite Aussagen zu den Rahmenbedingungen und Ausbildungsinhalten von Kinder- und Jugendlichenpsychotherapeuten erarbeitet wurden, werden hier Inhalte, die die spezifischen Versorgungsbedarfe von Kindern und Jugendlichen im Kontext künftiger Ausbildungs- 


\section{Editorial}

modelle in psychologischer Psychotherapie enthalten, weitestgehend ignoriert.

\section{Korrespondenzadresse}

Prof. Dr. J. M. Fegert

Klinik für Kinder- und Jugendpsychiatrie und

Psychotherapie, Universitätsklinikum UIm

Steinhövelstr. 5, 89075 Ulm, Deutschland

joerg.fegert@uniklinik-ulm.de

Interessenkonflikt. J.M. Fegert und H.J. Freyberge geben an, dass kein Interessenkonflikt besteht.

\section{Literatur}

Bundesministerium für Gesundheit (2016) Eckpunkte des Bundesministeriums für Gesundheit zur Novellierung der Ausbildung zum Psychologischen Psychotherapeuten. http://dgkjf. de/wp-content/uploads/BMG_PsychThG Eckpunkte-Direktausbildung.M.T.pdf. Zugegriffen: 14.12.2016

Fegert JM, Plener P, Freyberger HJ (2014) Psychotherapeutische Behandlungsansätze in der Adoleszenz. Psychotherapeut 59:5-6

Strauß B, Barnow S, Brähler E, Fegert JM, Fliegel S, Freyberger $\mathrm{HJ}$, Goldbeck L, Leuzinger-Bohleber M, Willutzki U (2009) Forschungsgutachten zur Ausbildung von Psychologischen PsychotherapeutInnen und Kinder- und JugendlichenpsychotherapeutInnen. Bundesministerium für Gesundheit, Berlin

\section{Schwerpunktthemen}

Das Herausgebergremium der Zeitschrift Psychotherapeut lädt Autorinnen und Autoren ein, an den geplanten Schwerpunkten mitzuarbeiten und geeignete Manuskripte einzureichen. Diese werden dem üblichen Reviewverfahren unterzogen. Darüber hinaus freuen wir uns über die Zusendung freier (unaufgeforderter) Originalia zu selbstgewählten Themen.

Bitte schicken Sie Ihren Beitrag an die Redaktion:

Regine.Karcher-Reiners@springer.com

Die Schwerpunktplanung ist vorläufig und kann kurzfristig umdisponiert werden.

Heft 4/2017: Psychotherapie mit Geflüchteten (Deadline 30.2.2017)

Heft 5/2017: Perspektiven hochschulbezogener Psychotherapie und Beratung (Deadline 30.4.2017)

Heft 6/2017: Psychotherapeutische Bezüge der Psychokardiologie (Deadline 30. 6. 2017)

Heft 1/2018: Herausforderungen und Möglichkeiten der Digitalisierung (Deadline 30.8.2017)

Heft 2/2018: Angst, Ressentiment, Hoffnung. Hass, Fanatismus, Versöhnung. Lindauer Psychotherapiewochen 2017 (Deadline 30.10.2017)

Heft 3/2018: Angst (Deadline 30.12.2017)

Heft 4/2018: Geschichtsvergessenheit (Deadline 30.2.2018)

Heft 5/2018: Sexualität und sexuelle Störungen (Deadline 30.4.2018)

Heft 6/2018: Studium zur Approbation (Deadline 30.6.2018) 